\title{
On Accelerating Stochastic Neural Networks
}

\author{
Swathika Ramakrishnan, Dhireesha Kudithipudi \\ NanoComputing Research Lab, Rochester Institute of Technology \\ Rochester, NY 14623
}

\begin{abstract}
Stochastic computing for neural networks is gaining traction for energy efficiency in neuromorphic systems. Generally, the accuracy of these systems is correlated with the the stochastic bit stream length and requires long compute times. In this study we propose methods to accelerate a stochastic computing based feedforward neural network, extreme learning machine. A new stochastic training hardware unit for the extreme learning machine is also proposed. In the proposed design a performance boost of $60.61 \mathrm{X}$ is achieved for Orthopedic dataset with $2^{12}$ bit stream length when tested on a Nvidia GeForce $1050 \mathrm{Ti}$. The design is also validated for two standardized datasets, an accuracy of $92.4 \%$ for MNIST dataset and $87.5 \%$ for orthopedic dataset is observed.
\end{abstract}

\section{KEYWORDS}

Stochastic computing, Neural Networks, neuromorphic, extreme learning machines

\section{ACM Reference format:}

Swathika Ramakrishnan, Dhireesha Kudithipudi. 2017. On Accelerating Stochastic Neural Networks. In Proceedings of NANOCOM '17, Washington D.C., DC, USA, September 27-29, 2017, 5 pages.

https://doi.org/10.1145/3109453.3123959

\section{INTRODUCTION}

Artificial Neural Networks (ANNs) have been widely used in various applications like gaming, speech recognition, mapping, etc. In recent years, there has been a growing interest in designing dedicated hardware substrates for these networks. The goal is to achieve energy efficiency, high network connectivity and better computational capabilities that are typically not optimized in the software ANN stack [12].

Stochastic computing is a natural choice to achieve complex arithmetic operations with basic logic gates and hereby reduce the total system energy [6]. For example a digital neuron consists of multiplication, summation and activation function as three major units. In a stochastic realization, they can be performed by simple circuits like AND gate, MUX operation and thresholding respectively. When implementing an 8-bit parallel weighted binary multiplier in stochastic logic an area reduction of $128 \mathrm{x}$ can be observed and a proportionate reduction in the power. Previous studies have

Permission to make digital or hard copies of all or part of this work for personal or classroom use is granted without fee provided that copies are not made or distributed for profit or commercial advantage and that copies bear this notice and the full citation on the first page. Copyrights for components of this work owned by others than ACM must be honored. Abstracting with credit is permitted. To copy otherwise, or republish to post on servers or to redistribute to lists, requires prior specific permission and/or a fee. Request permissions from permissions@acm.org.

NANOCOM '17, September 27-29, 2017, Washington D.C., DC, USA

(C) 2017 Association for Computing Machinery.

ACM ISBN 978-1-4503-4931-4/17/09 \$\$15.00

https://doi.org/10.1145/3109453.3123959 designed fpga-based stochastic neural network [2] and stochastic neural computation for a competitive learning network [4]. In all these instances, stochastic logic requires long computation time owing to the stochastic bit streams that are randomly generated. There is a correlation between the length of these bit streams and the accuracy of a task in a neural network [1].

The operation on these bits streams are bitwise and do not have inter dependencies. This offers a natural point to parallelize these operations to accelerate their compute time. Earlier research shows GPU implementation of Multilayer perceptron (MLP) [11], implementation of GMDH neural network [3], GPU implementation of spiking neural network for real-time systems [13] and mean-firing rate neural network implementation [5]. There is no specialized hardware developed exclusively for an ANN but the available resources can be adapted for the implementation of different applications [11]. This provides a two fold advantage ( $i$ ) rapid validation of large scale stochastic logic ANNs before custom-designing for hardware and (ii) to identify design knobs for performance boost. In this research, we perform a detailed analysis on a stochastic extreme learning machine. The stochastic bit stream generation, activation function, training unit, are identified as design knobs for GPU acceleration. A new stochastic training unit is proposed to efficiently realize the training of the output layer of an ELM. The overall speedup with GPU realization of the design is investigated at a block-level and network-scale. The design is validated on two standardized datasets, MNIST [8] and Orthopedic.

\section{STOCHASTIC LOGIC NEURAL NETWORKS}

In stochastic logic a signal is conveyed through the statistical distribution of the logical values and is represented by a random bit stream. The fractional numbers correspond to the probability of occurrence of a logical one versus a logical zero[6]. The value of the stochastic number is the probability of 1's in the bit stream. Mathematically, the stochastic numbers are represented by a bit-stream $\mathrm{X}$ with $\mathrm{N}$ bit length. There are two types of stochastic number representations [6], single line unipolar and single line bipolar representations. Throughout the work bipolar stochastic numbers are used for representing the stochastic bit streams as the ANN requires symmetric encoding.

\begin{tabular}{clc}
\hline Format & Number Value & Number Range \\
\hline \hline Unipolar & $N_{1} / \mathrm{N}$ & {$[0,1]$} \\
BiPolar & $\left(N_{1}-N_{0}\right) / \mathrm{N}$ & {$[-1,+1]$}
\end{tabular}

Table 1: Stochastic number representation with length of $\mathbf{N}, N_{1}$ 1's and $N_{0}$ o's 


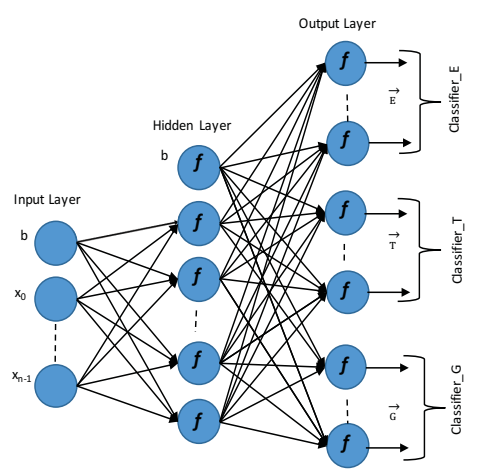

Figure 1: A Generic Structure of an ELM with multiple output layers

In a stochastic system both unipolar and bipolar can coexist. While generating stochastic bit streams, there might be correlation among numbers and the number can be altered by physical errors. This can be rectified by using a suitable stochastic number generator [1].

\subsection{Extreme Learning Machine}

Extreme learning machine (ELM), shown in Figure 1, is a feedforward neural network where the inputs are projected onto a single hidden layer with weights that are randomly initialized ( eg: bipolar weight ranges between $[-1,1])$. The hidden weights between the hidden layer and output layer are initialized randomly at the start and then updated at every epoch to arrive to the final weight values. The output layer performs either classification or regression tasks. Number of nodes in the input layer depends on the number of features and the hidden layer acts as an abstraction layer by making the inputs linearly independent of one another when projected on to a higher dimensional space. A unique feature of ELM is that the hidden layer is not trained and only the output layer neurons are trained. ELMs are shown to be faster than the traditional gradient-based learning algorithms [7]. In this work, ELM with online learning will be used.

Let us assume, there are $\mathrm{M}$ hidden layer nodes, number of input samples be $\mathrm{N}$ and the activation function is denoted by $\mathrm{g}(\mathrm{x})$. Let $\mathrm{H}$ be the matrix containing the output of hidden layer nodes, which is given by equation.

$$
H=h_{i} j=g\left(W_{j} \cdot X_{i}+b_{j}\right), \text { where } 1 \geq i \leq N \text { and } 1 \geq j \geq N
$$

where the product of weights $\left(W_{j}\right)$ and input matrix $\left(X_{i}\right)$ are summed with the bias value before feeding into the activation function. the output of the activation function is represented by $h_{i} j$. Output of the network is presented by $Y_{i}$ and the target output is $Y 1_{i}$.

$$
\begin{gathered}
\Delta W=\alpha\left(Y 1_{i}-Y_{i}\right) h_{i} j \\
g(x)=\frac{1}{1+e^{-x}}
\end{gathered}
$$

Sigmoid-like activation function is used in this work for the activation of the stochastic bit streams [2].

\section{ACCELERATING STOCHASTIC ELM IN GPU}

\section{Input Layer}

The input layer consists of the input nodes equivalent to the number of features. This layer can be accelerated by using two GPU blocks which generate the input and weight stochastic bit streams, (i) Decimal to Bipolar Stochastic Number converter (DBC) and (ii) Stochastic Number Generator (SNG). DBC block converts all the decimal numbers from $[-1,1]$ to $[0,1]$ range in 2 parallel operations by adding one to the decimal number in first step and then dividing it by 2 in the second parallel step. Relatively, a CPU will take $2 \mathrm{X}$ $\mathrm{M}$ steps where a $2 \mathrm{D}$ array is maintained to store the BSL values.

\section{Stochastic Number Generator}

SNG designed in this work is based on one of the standard methods, which compares the binary number with the random numbers to generate stochastic bits. To generate $\mathrm{M}$ stochastic numbers with $\mathrm{N}$ bits, it requires $M X N$ steps [1]. In this work, the above mentioned method is modified to work in one parallel step in GPU to obtain all the input nodes simultaneously by using a two level parallel SNG structure, the levels are (i) Generation of stochastic bits for all the input nodes in parallel and (ii) Generation of $\mathrm{N}$ stochastic bits for each of these input nodes in parallel as shown in the figure 2. Two inputs to the SNG are the BSL matrix and random number matrix. BSL matrix is converted to a $3 \mathrm{D}$ matrix of size $[M \times 1 \times N]$ by making $\mathrm{N}$ copies of the 2D BSL matrix and a random number matrix of same size with range $[0,1]$ has been generated. These two matrices are positionally mapped and given as an input to the SNG to generate an output matrix of same size. SNG consists of a comparator, which gives a 1 when the value in BSL matrix is greater than the random number matrix. The red boxes in the figure indicate the generation of stochastic bit stream for the first input node.

Totally three steps generate all the input nodes in the GPU, whereas it requires $(2 x M)+(M x N)=M(2+N)$ steps in the CPU. Next step in the network is to generate the synapses (weight values). In an ELM, similar to the input node generation, stochastic bit stream for weights can be generated in three parallel steps ( 2 for DBS and 1 for SNG) that is independent of the network size as shown in figure 3. In a CPU, this implementation will require $(2 x M x H)+$ $(M \times H \times N)=(M x H)(2+N)$ steps. Total number of computational steps for the input layer are 6 in the GPU and $M(2+N)(1+H)$ in the CPU.

\section{Hidden Layer}

Generation of Hidden layer nodes require two components, they are (i) Multiplier to find the product of the input and weight values for all the connections (synapse) and (ii) Activation function. Stochastic multiplier designed in this work has a three level parallel structure. They are (i) bitwise XNOR operation of input and weight stochastic bit streams (ii) stochastic multiplication of one input with all the weight values and (iii) multiplication of all the inputs and weights. Whole operation takes $(M x H x N)$ XNOR bitwise operations with no interdependencies. The input matrix from the input layer is 
converted from $[M x 1 \times N]$ to $[M x H x N]$ size by making $\mathrm{H}$ copies horizontally. This input matrix is positionally mapped with the Weight matrix of same size to carry out the parallel XNOR operation on both the matrix to obtain the product matrix in one parallel step.

\section{Activation Function}

The stochastic activation function designed in this work is based on the sigmoid like function. The input stochastic bit streams entering the hidden node are summed bit wise and compared with the threshold value equal to half of the total number of inputs. If the sum is greater than the threshold value, 1 is given as output otherwise a zero[2]. Total number of steps involved are $2 \mathrm{~N}$, which includes $\mathrm{N}$ summation and $\mathrm{N}$ comparison operations for $\mathrm{N}$ bits. The proposed design consists of a parallel structure which performs the summation and comparison in one parallel step. 3D product matrix of size $[M x H x N]$ is given to the GPU to give summation matrix of size $[1 x H x N]$. This matrix consists of values ranging from 0 to the number of hidden layers. This matrix is compared with the N/2 in one parallel to give the hidden layer output matrix. The activation function takes 2 steps to generate the hidden layer matrix from the product matrix. The total number of steps to generate the hidden nodes is four, where as in the CPU it is $(M x H x N)+2(H x N)$.

\section{Output Layer}

Output layer is initialized with random weights and then updated for each epoch. Stochastic number generator is used to generate these weights in one parallel step and followed by the GPU multiplier to get the stochastic bit streams for the output layer .

\section{Stochastic Training Circuit}

A novel GPU stochastic training circuit has been designed to perform the weight update operations as shown in Figure 7. This circuit is based on the delta update equation derived from the least mean squares [10]. $\Delta$ wi is the value by which the weight needs to be updated.

$$
\Delta w i=\left(y^{\prime(k)}-y(k)\right) h(m)
$$

The subtraction of the hidden layer output from the input labels uses a 2:1 MUX. Subtraction is achieved in parallel by using the MUX operation, as shown in equation 5; where $\mathrm{Z}$ is the output, $\mathrm{S}$ is the select line, $\mathrm{x}$ and $\mathrm{y}$ are the inputs. In oder to subtract two Stochastic numbers, one of the inputs need to be inverted. The whole process can be performed in three parallel steps: inversion, AND operation followed by an OR operation.

$$
\begin{aligned}
& Z=\left(x . S+y . S^{\prime}\right) \\
& Z=\left(x . S+y^{\prime} . S^{\prime}\right)
\end{aligned}
$$

In a CPU, $2(N x H)$ operations will be required for inversion and MUX operation of $\mathrm{H}$ hidden nodes with $\mathrm{N}$ stochastic bits.

The difference between the target and predicted value has to be multiplied by learning rate of the network alpha. It is the rate at which the network learns to update the synaptic weights through the iterations. To convert this alpha value to stochastic bit stream with good precision requires more number of bits throughout the system. To avoid this, a stochastic bit stream is converted

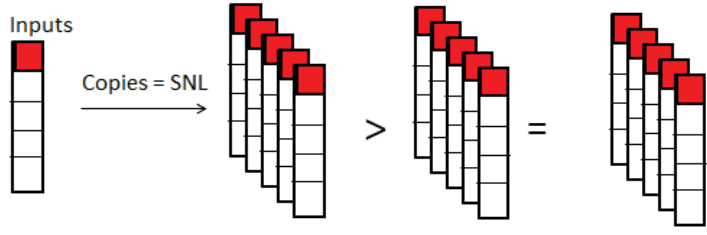

Figure 2: Visual representation of GPU implementation for the generation of input stochastic bit stream

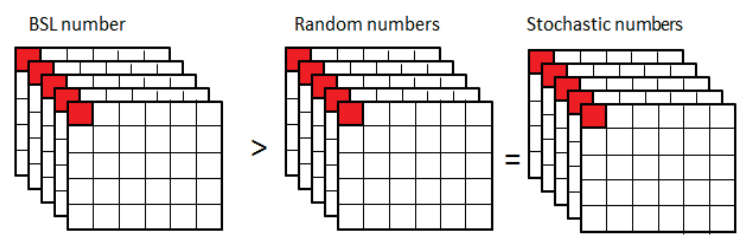

Figure 3: Visual representation of GPU implementation for the generation weights stochastic bit stream
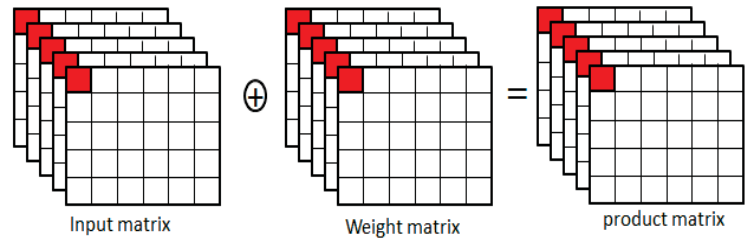

Figure 4: Visual representation of GPU stochastic multiplication using XNOR gate

to decimal number before multiplying with the alpha value. In figure 7, H2' represents the output of a multiplexer fed as input to the GPU stochastic to BSL conversion block (SBC). This block counts the number of 1's in each bit stream and divides the sum by SNL in to two parallel operations. BSL then converts to decimal number of range $[-1,1]$ using the BSL to decimal converter. All the elements of the output matrix from this block are multiplied by alpha value. Output of this block gives $\Delta \mathrm{W}$. The weights are updated by subtracting $\Delta \mathrm{W}$ from W2. Output of this block is converted back to BSL format and sent through SNG to get the stochastic bit streams for weights between the hidden and output layer. The total number of steps taken for training are eleven in a GPU. In a CPU the total number of steps will be, $(\mathrm{NxOL})+(\mathrm{NxOL})+(2 x \mathrm{OL})+(2 x \mathrm{OL})+\mathrm{OL}+\mathrm{W} 2+(2 x W 2)+(\mathrm{W} 2 x \mathrm{~N})$ $=(2 x O L)(N+2 O L+1 / 2)+W 2(1+2+N)$. The number of output nodes are represented by OL and the number of hidden layer weights by $\mathrm{W} 2$.

\section{RESULTS AND ANALYSIS}

For all the analysis, Nvidia GeForce 480s (1536 MB) and Nvidia GeForce $1050 \mathrm{Ti}$ are used for GPU implementation and Intel core-i5 processor is used for CPU implementation. This section talks about the regressive testing of network to narrow down the choice of parameter like Stochastic bit length and learing rate to achieve a good 


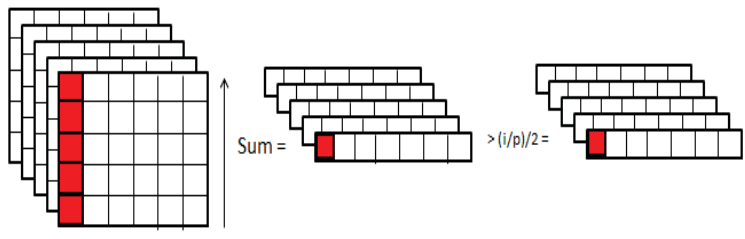

Figure 5: Visual representation of GPU implementation for Stochastic Sigmoid Activation Function

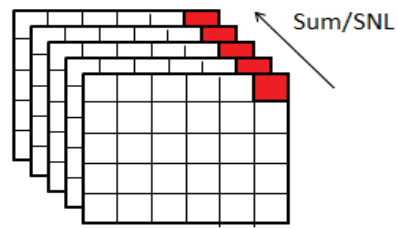

$=$

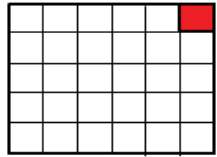

Figure 6: Visual representation of GPU implementation for stochastic to decimal conversion

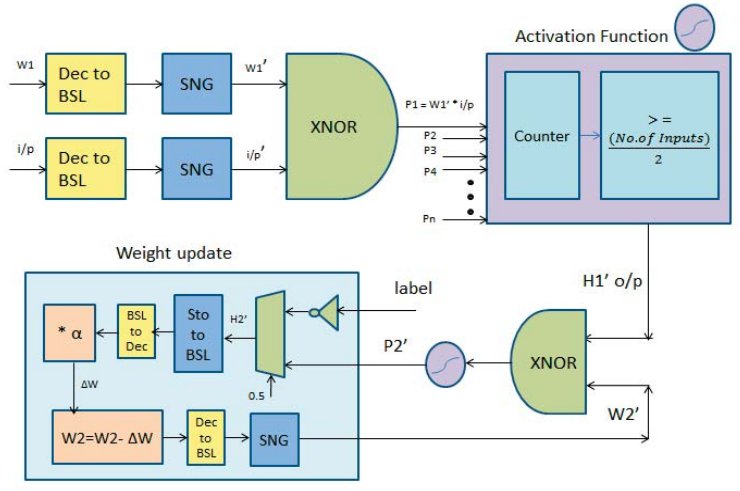

Figure 7: Stochastic Implementation of ELM

accuracy and also the performance boost achieved by implementing the network in two different GPUs and comparing its computation time with the CPU implementation.

\subsection{Choice of Parameters}

For a very small learning rate the ELM network trains very slow and takes time to reach a good accuracy. Similarly for large alpha, the learning curve of the network will contain undulations and will not be exponential. Figure 9 shows the accuracy plots for different alpha values for MNIST and Orthopedic datasets[9]. This shows that accuracy increases with increase in alpha value from 0.0001 to 0.1 and saturates after that in the case of the MNIST dataset. In the case of the orthopedic dataset a good accuracy is achieved below 0.001 .

In order to choose the appropriate bit length, mean square error (MSE) and mean absolute error (MAE) are studied for different bit lengths. Mean square error is a plot of average of the square of the difference between the target and the predicted output. Figure

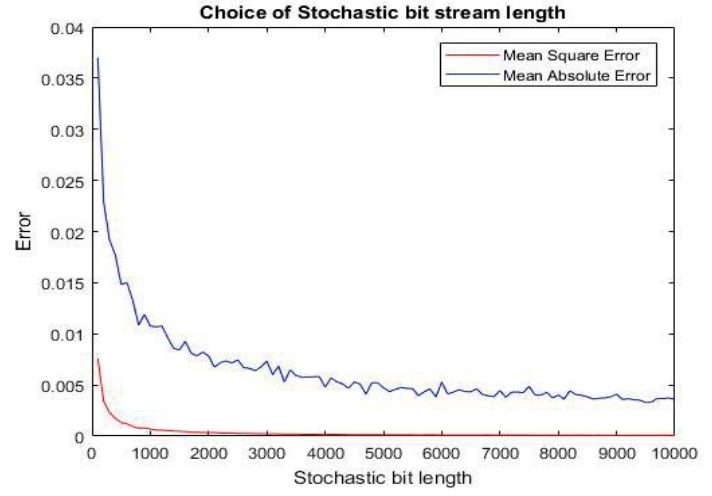

Figure 8: Stochastic bit length vs. MSE for MNIST dataset

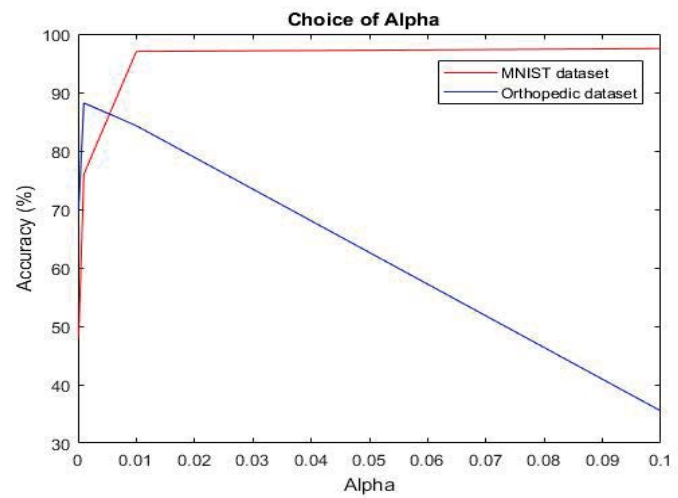

Figure 9: Stochastic ELM Accuracy Vs Alpha for MNIST and Orthopedic datasets

10 shows that increased bit length mitigates this problem. It is well understood that as the bit length increases stochastic numbers can be represented with better precision. Figure 8 also gives the absolute error, absolute value of the difference between the target and predicted against increasing bit length. For a bit size of 2000 the error caused due to bit generation is around 0.0055 whereas it is 0.01 for the arithmetic blocks. Around $2^{12}$ bit stream length, the mean absolute error is 0.05 . We have used this bit stream length for rest of the analysis.

When the ELM is tested with the Orthopedic dataset a maximum accuracy of $90.2 \%$ is achieved, with 200 hidden nodes and an alpha value of 0.001 . The stochastic GPU implementation of the same network gives a test accuracy of $87.5 \%$ as shown in Figure 10, with an accuracy drop of around $2 \%$. One major contributor to the accuracy drop is the subtraction block in the training unit of the stochastic ELM, where only half of the sum of two inputs is obtained. In some scenarios, where resource utilization is of prime concern, a $2 \%$ drop in accuracy might be tolerable. Figure 10 shows the accuracy for MNIST data set, with 0.01 alpha value. 


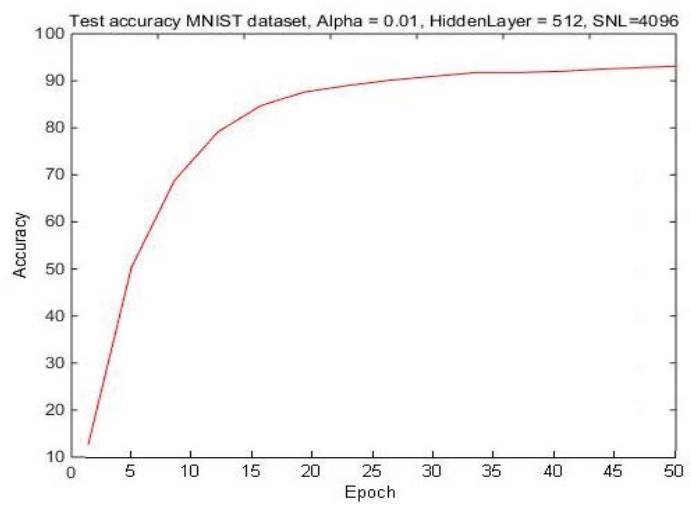

Figure 10: Stochastic ELM accuracy with MNIST dataset, Alpha $=0.01$

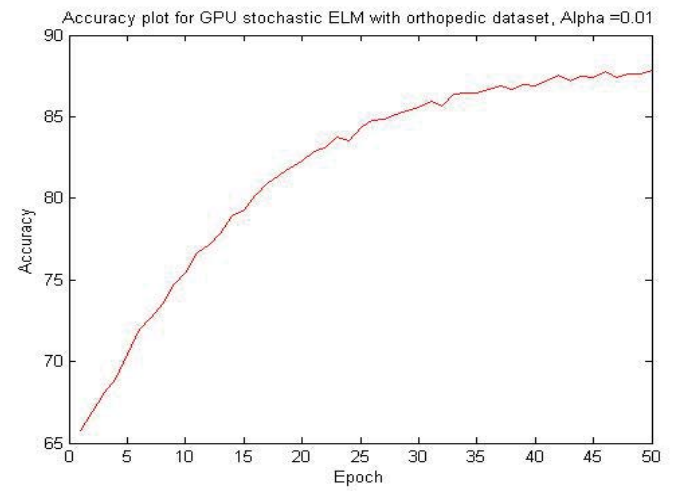

Figure 11: Stochastic ELM accuracy with Orthopedic dataset,Alpha $=0.001$

\subsection{Speedup}

The stochastic ELM is tested on two different GPUs. They are (i) GeForce GTX 480s Nvidia graphics card which has 480 cores, 2.0 CUDA capability and $1538 \mathrm{MB}$ memory configuration and (ii) NVIDIA GeForce GTX 1050 Ti with 4GB memory with 768 cores and 6.1 CUDA capability. The timing results in table 2 and 3 for Orthopedic dataset shows that the increase in computation time is relatively low compared to the increase in bit length, thus proving that GPU implementation works better than in CPU for higher bit streams. Among the two, the difference in speedup with the increase in bit stream decreases for the lower version GPU and increases for the other, this is because GTX 480s reaches the hardware limit for large bit streams.

\section{CONCLUSIONS}

This work shows a GPU realization of a stochastic ELM for rapid prototyping. The design tested on various parameters like learning rate, bit length and number of hidden layers quickly narrows down on the best hardware realization. By using a hybrid stochasticdeterministic ELM design, the overall performance can be further

\begin{tabular}{cccc}
\hline SNL & CPU (mins) & GeForce GTX 480s (mins) & Speedup \\
\hline \hline $2^{11}$ & 1842.34 & 132.3 & 13.93 \\
$2^{12}$ & 3661.42 & 204.2 & 17.93 \\
$2^{13}$ & 7242.76 & 384 & 18.86
\end{tabular}

Table 2: Speedup of the Stochastic ELM network size $6 \mathrm{X} 512 \mathrm{X} 2$ tested on orthopedic dataset over 50 epochs on GeForce GTX 480s GPU

\begin{tabular}{llcl}
\hline SNL & CPU (mins) & GeForce GTX 1050 Ti (mins) & Speedup \\
\hline \hline $2^{11}$ & 1842.34 & 51.2 & 35.99 \\
$2^{12}$ & 3661.42 & 60.4 & 60.61 \\
$2^{13}$ & 7242.76 & 68.8 & 105.57 \\
\hline
\end{tabular}

Table 3: Speedup of the Stochastic ELM network size 6X512X2 tested on orthopedic dataset over 50 epochs on GeForce GTX 1050 Ti GPU

improved. Non-complex arithmetic blocks like adders can be realized in the deterministic domain and the complex blocks can be realized in the non-deterministic domain.

\section{REFERENCES}

[1] Armin Alaghi and John P. Hayes. 2013. Survey of Stochastic Computing. ACM Trans. Embed. Comput. Syst. 12, 2s, Article 92 (May 2013), 19 pages. https: //doi.org/10.1145/2465787.2465794

[2] S. L. Bade and B. L. Hutchings. 1994. FPGA-based stochastic neural networksimplementation. In FPGAs for Custom Computing Machines, 1994. Proceedings. IEEE Workshop on. 189-198. https://doi.org/10.1109/FPGA.1994.315612

[3] Ricardo Brito, Simon Fong, Kyungeun Cho, Wei Song, Raymond Wong, Sabah Mohammed, and Jinan Fiaidhi. 2016. Towards implementation of residual-feedback GMDH neural network on parallel GPU memory guided by a regression curve. The fournal of Supercomputing 72, 10 (2016), 3993-4020.

[4] B. D. Brown and H. C. Card. 2001. Stochastic neural computation. II. Soft competitive learning. IEEE Trans. Comput. 50, 9 (Sep 2001), 906-920. https: //doi.org/10.1109/12.954506

[5] Helge ÃIJ. Dinkelbach, Julien Vitay, Frederik Beuth, and Fred H. Hamker. 2012. Comparison of GPU- and CPU-implementations of mean-firing rate neural networks on parallel hardware. Network (Bristol, England) 23, 4 (2012), 212.

[6] B. R. Gaines. 1969. Stochastic Computing Systems. Springer US, Boston, MA, 37-172. https://doi.org/10.1007/978-1-4899-5841-9_2

[7] Guang-Bin Huang, Dian Hui Wang, and Yuan Lan. 2011. Extreme learning machines: a survey. International fournal of Machine Learning and Cybernetics 2, 2 (2011), 107-122. https://doi.org/10.1007/s13042-011-0019-y

[8] Yann LeCun, Léon Bottou, Yoshua Bengio, and Patrick Haffner. 1998. Gradientbased learning applied to document recognition. Proc. IEEE 86, 11 (1998), 22782324

[9] M. Lichman. 2013. UCI Machine Learning Repository. (2013). http://archive.ics. uci.edu/ml

[10] C. Merkel and D. Kudithipudi. 2014. A stochastic learning algorithm for neuromemristive systems. In 201427 th IEEE International System-on-Chip Conference (SOCC). 359-364. https://doi.org/10.1109/SOCC.2014.6948954

11] Kyoung-Su Oh and Keechul Jung. 2004. \{GPU\} implementation of neural networks. Pattern Recognition 37, 6 (2004), 1311 - 1314. https://doi.org/10.1016/j. patcog.2004.01.013

[12] E. Won. 2007. A hardware implementation of artificial neural networks using field programmable gate arrays. Nuclear Instruments and Methods in Physics Research Section A: Accelerators, Spectrometers, Detectors and Associated Equipment 581, 3 (2007), 816 - 820. https://doi.org/10.1016/j.nima.2007.08.163

[13] Dmitri Yudanov. 2009. GPU-based implementation of real-time system for spiking neural networks. (2009). 